\title{
Preparation of perfluoroalkane-tagged chiral auxiliaries and their application to stereoselective synthesis of a $\beta^{2}$-amino acid building block
}

\author{
Markus M. Vögtle, * Daniel A. S. Beck, Thomas Leutert, \\ Flavio Ossola, and Luigi La Vecchia \\ Novartis Institutes for BioMedical Research, Novartis Pharma AG, CH-4057 Basel \\ markus.voegtle@novartis.com
}

\begin{abstract}
Both enantiomers of novel perfluoroalkane-tagged 5,5-diphenyl-2-oxazolidinone (DIOZ) auxiliaries have been prepared using a two-step one-pot synthesis, starting from commercially available $N$-(tert-butoxycarbonyl)- $L$-valine methyl ester. The key feature of this approach is the efficient generation of a suitably active perfluoroalkyl-aryllithium species. By use of this protocol, the perfluoroalkane-tagged DIOZ auxiliaries are obtained in high enantiomeric purity and on multigram scales with overall yields exceeding $50 \%$. The perfluoroalkane-tagged auxiliaries enable the use of fluorous solid-phase extraction, allowing efficient purification of tagged intermediates from crude reaction mixtures. The new auxiliaries have been applied in an asymmetric synthesis of $\beta^{2}$-N-Fmoc-phenylalanine via a stereoselective conjugate addition.
\end{abstract}

Keywords: Fluorous phase, solution-phase synthesis, separation, $\beta^{2}$-amino acid

\section{Introduction}

In the field of stoichiometric asymmetric synthesis, fluorous solid-phase extraction (FSPE) techniques have been applied to many chemical transformations, offering a powerful alternative to standard separation and purification methods. Initially proposed as a method of separating catalysts from process streams, perfluoroalkane chains have been developed as general supports for organic reagents and auxiliaries. Perfluoroalkane-tagged chiral auxiliaries are soluble in typical organic reaction solvents, and the perfluoroalkane chains are impervious to most common reagents.

Generally, minor or no modifications to the classical reaction conditions are necessary. After a reaction is complete, selective recovery of the tagged material is easily accomplished by FSPE. Several research groups from academia and industry have demonstrated that fluorous synthesis 
with chiral auxiliary methods is both viable and broadly applicable. For example, fluorous Evans-auxiliaries have been synthesized and applied successfully in aldol reactions and conjugate radical additions. ${ }^{1-3}$ In this paper we report the short and efficient synthesis of perfluoroalkane tagged chiral DIOZ auxiliaries and their application to a novel synthesis of $\beta^{2}$ phenylalanine. $^{4-6}$

\section{Results and Discussion}

Our initial approach to the synthesis was aimed at a late stage introduction of the perfluoroalkane tag to enable the use of a common intermediate in the preparation of a diverse range of auxiliaries in terms of the perfluoroalkane chain length and the manner of attachment of the tag (Scheme 1).

The oxazolidinone 3a was synthesized using a modification of the procedure described by Seebach. ${ }^{4}$ para-Bromophenol 1a was protected with TIPS or TBDMS groups, via a standard protocol (silyl chloride, imidazole) to provide compounds $\mathbf{1 b}$ and $\mathbf{1 c}$ respectively, in nearly quantitative yields. The protected phenols were then converted into the corresponding Grignard reagents. The thus formed organo-magnesium halide species were immediately reacted with $N$ (tert-butoxycarbonyl)- $L$-valine methyl ester to deliver the tertiary alcohol intermediates $\mathbf{2 b}$ and 2c in good yields (87-95\%). Treatment of these open-chain intermediates with potassium tertbutoxide in THF at room temperature gave the oxazolidinones $\mathbf{3 b}$ and $\mathbf{3 c}$ in $81 \%$ and $78 \%$ yields, respectively.

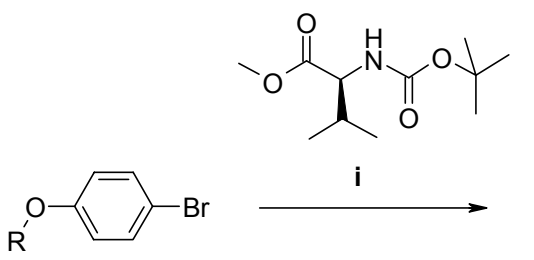

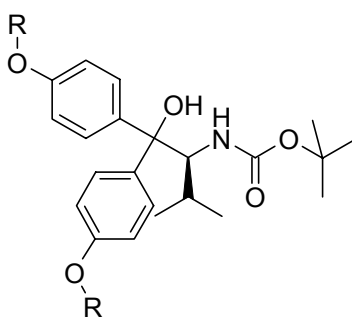

2

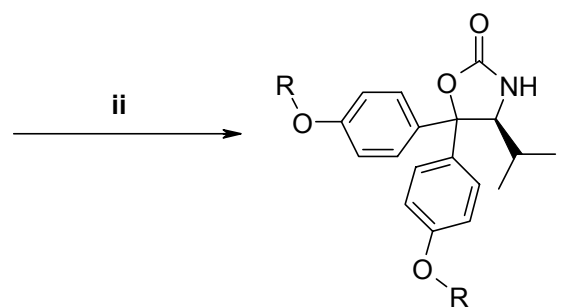

3

(i) $\mathrm{Mg}, \mathrm{THF}, 17 \mathrm{~h}, 0{ }^{\circ} \mathrm{C} \rightarrow \mathrm{RT}, 87-95 \%$. (ii) $\mathrm{KO}^{\mathrm{t}} \mathrm{Bu}, \mathrm{THF}, 16 \mathrm{~h}, 0{ }^{\circ} \mathrm{C}-\mathrm{RT}, 78-81 \%$.

Scheme 1. Synthesis of Oxazolidinones. 
Deprotection with HF•pyridine afforded the free bis-phenol 3a (84-90\%), which could be converted into the desired perfluoroalkane-tagged DIOZ auxiliaries either by re-protection with a freshly prepared perfluoroalkylsilyl chloride affording DIOZ 3d (59\%) or alkylation with hexadecafluoro-nonane triflate to provide perfluoro-ether DIOZ 3e (43\%).

Table 1. Derivatives of $\mathbf{1 , 2}$ and 3

\begin{tabular}{llllll}
\hline & $\mathrm{R}$ & & $\mathrm{R}$ & & $\mathrm{R}$ \\
\hline $\mathbf{1 a}$ & $\mathrm{H}$ & & & $\mathbf{3 a}$ & $\mathrm{H}$ \\
$\mathbf{1 b}$ & TIPS & $\mathbf{2 b}$ & TIPS & $\mathbf{3 b}$ & TIPS \\
$\mathbf{1 c}$ & TBDMS & $\mathbf{2 c}$ & TBDMS & $\mathbf{3 c}$ & TBDMS \\
& & & & $\mathbf{3 d}$ & $\left.\mathrm{Si}\left(\mathrm{CH}_{(\mathrm{CH}}\right)_{2}\right)_{2} \mathrm{CH}_{2} \mathrm{CH}_{2} \mathrm{C}_{8} \mathrm{~F}_{17}$ \\
& & & $\mathbf{3 e}$ & $\mathrm{CH}_{2}\left(\mathrm{CF}_{2}\right)_{7} \mathrm{CF}_{2} \mathrm{H}$ \\
\hline
\end{tabular}

An alternative DIOZ auxiliary, where attachment of the perfluoroalkane tag to the phenyl rings is via a carbon-carbon bond, was synthesized from the commercially available perfluoroalkylaryl bromide $4\left(\mathrm{R}^{\mathrm{f}}=\mathrm{C}_{2} \mathrm{H}_{4} \mathrm{C}_{6} \mathrm{~F}_{13}\right.$, Scheme 2). Compound 4 was treated with $n$-BuLi at low temperature to afford the corresponding metallated species and immediately allowed to react with $N$-(tert-butoxycarbonyl)- $L$-valine methyl ester, providing the tertiary alcohol $5(82 \%)$. Surprisingly, the application of the Grignard protocol resulted in absolutely no conversion, presumably as a result of failure to initiate formation of the Grignard reagent. Treatment of the perfluoroalkane-tagged open-chain intermediate $\mathbf{5}$ with potassium tert-butoxide in THF gave the DIOZ compound 6 in 57\% yield. A more expeditious version of this route involved the synthesis of DIOZ auxiliary 6 from the commercially available aryl bromide 4 via metallation, addition and cyclisation in a one-pot process. The overall yield using this most convenient route was $50 \%$. Using the same one-pot protocol but starting with $N$-(tert-butoxycarbonyl)- $D$-valine methyl ester, afforded the optical antipode of the auxiliary, namely DIOZ ent-6.

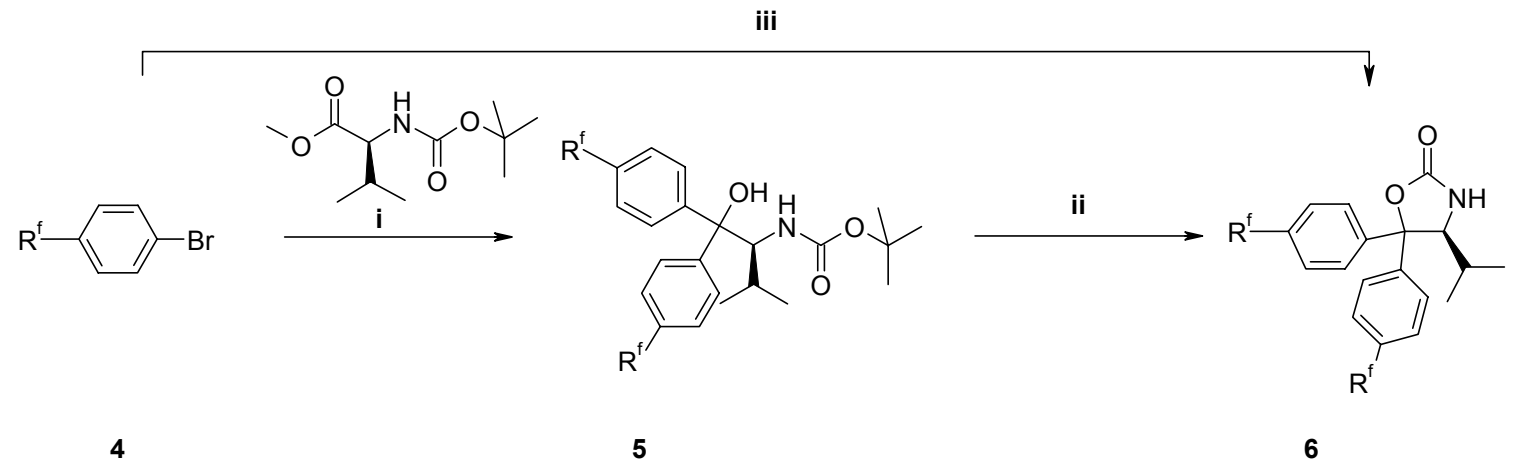

Scheme 2. Synthesis of perfluoroalkane tagged DIOZ auxiliary $6\left(\mathrm{R}^{\mathrm{f}}=\mathrm{C}_{2} \mathrm{H}_{4} \mathrm{C}_{6} \mathrm{~F}_{13}\right)$.

(i) $n$-BuLi, THF, $-78{ }^{\circ} \mathrm{C}, 6 \mathrm{~h}, 82 \%$. (ii) $\mathrm{KO} t$-Bu, THF, $18 \mathrm{~h}, 0{ }^{\circ} \mathrm{C}-\mathrm{RT}, 57 \%$. (iii) $n$-BuLi, THF, $78^{\circ} \mathrm{C}, 6 \mathrm{~h}$, then $\uparrow 18{ }^{\circ} \mathrm{C}, 2 \mathrm{~h}, 50 \%$. 
The novel perfluoroalkane tagged chiral auxiliaries were then applied to stereoselective syntheses of a $\beta^{2}$-amino acid as depicted in Scheme 3.,7 A key aspect of this protocol was the application of FSPE purification techniques. As the fluorous tagged chiral auxiliaries $\mathbf{3 d}$, 3e and 6 showed similar chromatographic behavior in several elution and separation experiments we choose $\mathbf{6}$ and ent-6 as auxiliaries in an asymmetric synthesis on larger scale due to the ease of synthesis. Both enantiomers of the required $\alpha, \beta$-unsaturated imide starting material, 7 and ent-7, were prepared using a standard acylation protocol (DMAP, DIPCD, 2 eq. 2-benzylacrylic acid) in good yield (78\%). The imide intermediates 7 and ent-7 were exposed to a twofold excess of $O$-benzyl hydroxylamine at $72{ }^{\circ} \mathrm{C}$ for $24 \mathrm{~h}$ in the key stereoselective conjugate addition reaction. Lower temperatures required significantly longer reaction times. The resultant aza-Michael adducts 8 and ent-8 were purified using FSPE: A standard glass flash chromatography column was packed with commercially available Fluoroflash ${ }^{\mathrm{TM}}$ and pre-conditioned by passing through 80:20 MeOH $-\mathrm{H}_{2} \mathrm{O}(\mathrm{v} / \mathrm{v})$ under positive pressure. The residue was loaded on to the top of the column, using DMF as the loading solvent. The column was then eluted with $\mathrm{MeOH} / \mathrm{H}_{2} \mathrm{O}$. The resulting crude products $\mathbf{8}$ and ent-8 were a $c a$. 8.3:1 mixture of diastereomers, judged by HPLC, at the stereogenic centre introduced by the conjugate addition reaction. Formation of the tosylate salt of compound $\mathbf{8}$ and subsequent recrystallization thereof, improved the diastereomeric excess to $93 \%$ as determined by ${ }^{1} \mathrm{H}-\mathrm{NMR}$ and HPLC. Removal of the auxiliary $\mathbf{6}$ and concomitant release of the $\beta^{2}$-aminoxy acid 9 was facilitated with $\mathrm{LiOH} / \mathrm{H}_{2} \mathrm{O}_{2}$ in $\mathrm{THF} / \mathrm{H}_{2} \mathrm{O}(74 \%)$. The auxiliary was conveniently recovered $(96 \%)$ using the same FSPE procedure. The previously reported $\beta^{2}$-aminoxy acid 9 was converted to the corresponding $\beta^{2}$-amino acid $\mathbf{1 0}$ via hydrogenation $\left(\mathrm{H}_{2}, \mathrm{Pd} / \mathrm{C}, \mathrm{MeOH}, \mathrm{NH}_{3}, \mathrm{H}_{2} \mathrm{O}\right)$ and Fmoc-protected using standard conditions (FmocOSu, $\mathrm{NaHCO}_{3}, \mathrm{THF}$ ) to afford compound 11 in 30\% over the two steps.

This sample of the Fmoc- $\beta^{2}$-amino acid $\mathbf{1 1}$ was identical, as judged by ${ }^{1} \mathrm{H}-\mathrm{NMR}$ and low resolution mass spectrometric analysis, to previously reported authentic material. ${ }^{8,9}$ The depicted absolute stereochemistry is correlated to the chiral HPLC retention characteristics of the authentic material. The enantiomeric excess of the Fmoc- $\beta^{2}$-amino acid 11 resulting from both isomers of the auxiliary was analyzed via HPLC and found to exceed 95\%. 

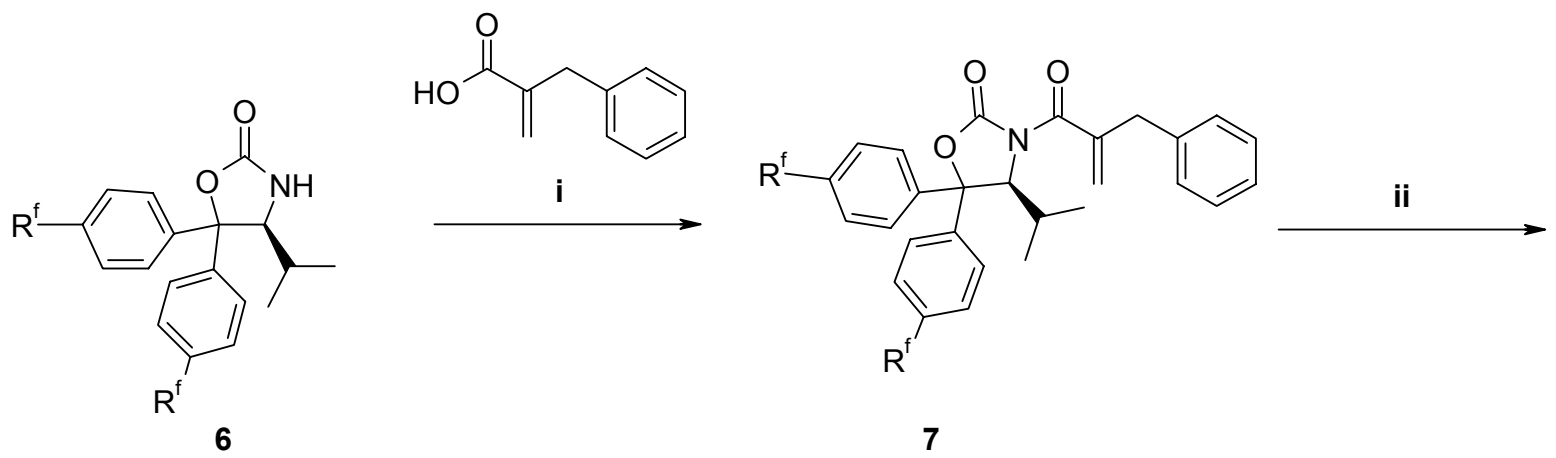<smiles>[R]c1ccc(C2(c3ccc([R])cc3)OC(=O)N(C(=O)[C@H](CNOCc3ccccc3)Cc3ccccc3)[C@H]2C(C)C)cc1</smiles>

8

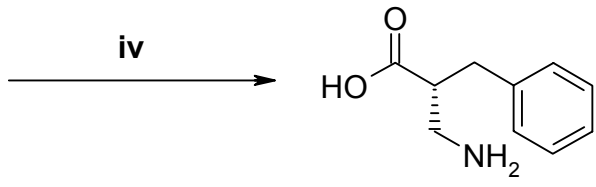

10

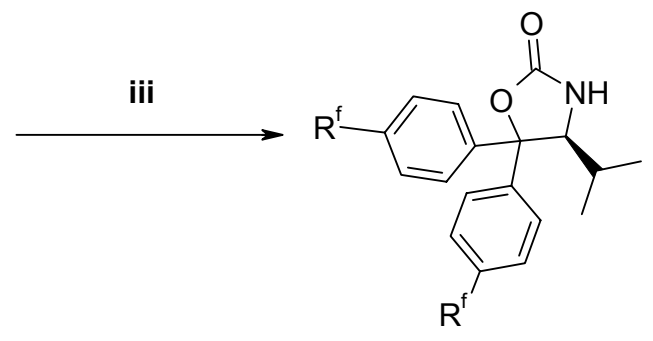

6<smiles>O=C(O)[C@H](CNOCc1ccccc1)Cc1ccccc1</smiles>

9

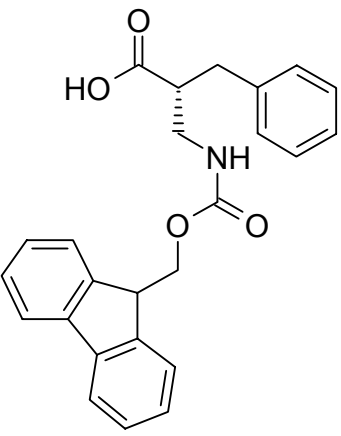

11

(i) DMAP, DIPCD, DCM, $0{ }^{\circ} \mathrm{C}$ - RT 16 h 78\%. (ii) $O$-benzyl hydroxylamine, THF, $72{ }^{\circ} \mathrm{C}, 24 \mathrm{~h}$, $70 \%$. (iii) $\mathrm{H}_{2} \mathrm{O}_{2}, \mathrm{LiOH}, \mathrm{THF}, \mathrm{H}_{2} \mathrm{O}, 45 \mathrm{~min}, 74 \%$. (iv) $\mathrm{H}_{2}, \mathrm{Pd} / \mathrm{C}(10 \%), \mathrm{MeOH}, \mathrm{H}_{2} \mathrm{O}, \mathrm{NH}_{3}$ (v) Fmoc-OSu, $\mathrm{NaHCO}_{3}$, THF, $\mathrm{H}_{2} \mathrm{O}, 0{ }^{\circ} \mathrm{C} \rightarrow \mathrm{RT}, 2 \mathrm{~h}, 30 \%$ over two steps.

Scheme 3. Synthesis of Fmoc- $\beta^{2}$-phenylalanine using FSPE.

\section{Conclusions}

The experiments described in this publication have enabled production of perfluoroalkane-tagged DIOZ chiral auxiliaries from commercially available starting materials in a one-pot operation. Application of the perfluoroalkane-tagged DIOZ auxiliaries provided a short and efficient 
synthesis of both enantiomeric forms of $\beta^{2}-N$-Fmoc-phenylalanine (described in the Experimental Section). ${ }^{14,15}$ This route is distinguished by its succinctness and ease of recovery of the DIOZ auxiliary. Purification of products was achieved through silica and fluorous solidphase extractions, with only one recrystallization being required. We have performed the complete sequence on various scales, up to the formation of $25 \mathrm{~g}$ of the target compound in a single batch and the process requires no equipment other than typical laboratory glassware. We believe that the reported perfluoroalkane-tagged DIOZ auxiliaries are truly powerful tools for drug discovery and high-throughput chemistry and are viable alternatives to conventional or solid supported auxiliaries.

\section{Experimental Section}

General Procedures. All reagents were obtained commercially and used as received unless otherwise noted. TLC was performed on Merck silica gel plates 60 F-254, Art. no. 5729.

Reverse-phase HPLC analyses were performed on an Agilent-1100 using a Macherey-Nagel CC 70/4 Nucleosil 100-3 C18 HD column, acetonitrile and water both containing 0.05\% TFA, a column temperature of $35{ }^{\circ} \mathrm{C}$, with a flow rate of $1.0 \mathrm{~mL} / \mathrm{min}$ and measuring at $216 \mathrm{~nm}$. The standard gradient used was $5-100 \% \mathrm{MeCN}$ over $6 \mathrm{~min}, 100 \% \mathrm{MeCN}$ for $1.5 \mathrm{~min}$ followed by $100-5 \% \mathrm{MeCN}$ over $0.5 \mathrm{~min}$.

NMR was performed using a $400 \mathrm{MHz}$ Varian spectrometer, AS 400 Oxford. ${ }^{1} \mathrm{H}$ shifts were referenced to $\mathrm{CDCl}_{3}$ at $7.25 \mathrm{ppm}$ with tetramethylsilane as internal standard for ${ }^{1} \mathrm{H}$ NMR. MS was measured using VG Platform (Fisons Instruments), Spectraflow 783 Detector, HP 1100 Series HPLC. Melting points were measured using a Büchi, B-545 apparatus.

(4-Bromo-phenoxy)triisopropylsilane (1b). A solution of 4-bromophenol (1a, $43.96 \mathrm{~g}$, $254 \mathrm{mmol})$ and TIPS-Cl (54.95 mL, $257 \mathrm{mmol})$ in DCM, was cooled, on an ice bath to $0{ }^{\circ} \mathrm{C}$ whilst being stirred under nitrogen. The solution was treated with imidazole $(43.3 \mathrm{~g}, 636 \mathrm{mmol})$ added gradually with caution, and then allowed to warm to RT, with stirring, over the course of $16 \mathrm{~h}$. The resulting suspension was washed sequentially with $\mathrm{HCl}(2 \times 200 \mathrm{~mL}$ of a $0.5 \mathrm{M}$ aqueous solution), sat. aq. $\mathrm{NaHCO}_{3}(200 \mathrm{~mL})$ and brine $(200 \mathrm{~mL})$, then dried $\left(\mathrm{MgSO}_{4}\right)$ and concentrated under reduced pressure. The resulting residue was taken up into hexane $(400 \mathrm{~mL})$ and adsorbed onto silica gel (200 g) under reduced pressure. The resulting free-flowing solid was then added to the top of a short plug of silica $(70 \mathrm{~g})$ and eluted with hexane $(3000 \mathrm{~mL})$. Concentration of the appropriate fractions $\left(R_{\mathrm{f}}=0.6-0.8\right)$ under reduced pressure afforded compound $\mathbf{1 b}(81.7 \mathrm{~g}, 248 \mathrm{mmol}, 98 \%)$ as a colourless oil.

${ }^{1} \mathrm{H}-\mathrm{NMR}\left(400 \mathrm{MHz}, \mathrm{CDCl}_{3}\right) \delta$ 7.31(2H, m), $6.76(2 \mathrm{H}, \mathrm{m}), 1.24(3 \mathrm{H}, \mathrm{m}), 1.09(18 \mathrm{H}, \mathrm{d}, J=8.0$ $\mathrm{Hz}) .{ }^{13} \mathrm{C}-\mathrm{NMR}\left(100 \mathrm{MHz}, \mathrm{CDCl}_{3}\right) \delta 155.5,132.5,121.9,113.4,18.1,12.8$.

Mass Spectrum (ESI, -MS) m/z 327 (M-H), 249 (M-HBr). 
(4-Bromo-phenoxy)-tert-butyldimethylsilane (1c). Compound 1c was prepared in an analogous fashion to the preparation of compound 1b, using 4-bromophenol (1a, $33.7 \mathrm{~g}, 194.8 \mathrm{mmol}$ ) as starting material. Purification via flash chromatography $\left(R_{\mathrm{f}}=0.5-0.7\right.$, hexane elution $)$ afforded compound 1c (53.73 g, $187 \mathrm{mmol}, 96 \%$ ) as a colourless oil. ${ }^{1} \mathrm{H}-\mathrm{NMR}\left(400 \mathrm{MHz}, \mathrm{CDCl}_{3}\right) \delta 7.33$ $(2 \mathrm{H}, \mathrm{m}), 6.73(2 \mathrm{H}, \mathrm{m}), 0.99(9 \mathrm{H}, \mathrm{s}), 0.20(6 \mathrm{H}, \mathrm{s})$.

(-)-\{(S)-1-[Hydroxy-bis-(4-triisopropylsilanyloxyphenyl)methyl]-2-methyl-propyl $\}$ carbamic acid tert-butyl ester (2b). The following protocol was adapted from a previously reported procedure. ${ }^{4}$ A dry $250 \mathrm{~mL}$ round-bottomed flask fitted with a condenser, dropping funnel, thermometer and magnetic stirrer, was charged with Mg turnings (6.42 g, $264 \mathrm{mmol})$ and stirred, dry, under an atmosphere of nitrogen, overnight. A solution of compound $\mathbf{1 b}(8.0 \mathrm{~g}, 24.3 \mathrm{mmol})$ in THF $(20 \mathrm{~mL})$ was added and stirring continued, until the reaction started spontaneously as evidenced by refluxing of the THF. At this stage, more of compound $\mathbf{1 b}$ (74.82 $\mathrm{g}, 227.2 \mathrm{mmol}$ ) in THF $(60 \mathrm{~mL})$ was added, dropwise, at a rate sufficient to maintain reflux. The resultant mixture was heated at reflux, for $2 \mathrm{~h}$, then cooled to below $6^{\circ} \mathrm{C}$ on an ice bath. A solution of $N$ (tert-butoxycarbonyl)- $L$-valine methyl ester (17.46 g, $75.5 \mathrm{mmol})$ in THF $(20 \mathrm{~mL})$ was added dropwise, at such a rate as to maintain the internal reaction temperature below $6{ }^{\circ} \mathrm{C}$, then the mixture was allowed to warm to RT, with stirring, over $18 \mathrm{~h}$. The mixture was poured into icecold sat. aq. $\mathrm{NH}_{4} \mathrm{Cl}$ (200), the aqueous phase was separated and extracted with EtOAc (2 x 150 $\mathrm{mL})$. The combined organic phases were washed with brine $(150 \mathrm{~mL})$ and dried $\left(\mathrm{MgSO}_{4}\right)$, concentrated under reduced pressure and purified via flash chromatography. Concentration of the appropriate fractions $\left(R_{\mathrm{f}}=0.3,95: 5\right.$ hexane / EtOAc) gave compound $\mathbf{2 b}$ (44 g, $65.85 \mathrm{mmol}$, 87\%). ${ }^{1} \mathrm{H}-\mathrm{NMR}\left(400 \mathrm{MHz}, \mathrm{CDCl}_{3}\right) \delta$ 7.33-7.21 (4H, m), 6.80-6.75 (4H, m), $5.00(1 \mathrm{H} \mathrm{d}, J=12$ $\mathrm{Hz}), 4.45(1 \mathrm{H}, \mathrm{m}), 2.49(1 \mathrm{H}, \mathrm{s}), 1.78(1 \mathrm{H}$, complex m), $1.44(1 \mathrm{H}$, br. s), $1.36(9 \mathrm{H}, \mathrm{s}), 1.21(6 \mathrm{H}$, complex m), $1.06(36 \mathrm{H}, \mathrm{d}, J=8.0 \mathrm{~Hz}), 0.86(3 \mathrm{H}, \mathrm{d}, J=8.0 \mathrm{~Hz}), 0.81(3 \mathrm{H}, \mathrm{d}, J=8.0 \mathrm{~Hz}) .{ }^{13} \mathrm{C}-$ NMR $\left(100 \mathrm{MHz}, \mathrm{CDCl}_{3}\right) \delta 156.5,154.8,139.5,138.6,127.2,126.8,119.8,119.6,82.1,79.1$, 59.4, 28.9, 28.6, 22.9, 18.1, 17.6, 12.9, 12.8. Mass Spectrum (ESI, -MS) $m / z 698$ (M-H), 249 (TIPS-Phenol -H). Specific Rotation $[\alpha]_{\mathrm{D}}{ }^{2{ }^{\circ} \mathrm{C}}-39$ (c 1.0, $\left.\mathrm{MeOH}\right)$. HRMS (ESI $\left.{ }^{+}\right)$calcd for $\mathrm{C}_{40} \mathrm{H}_{69} \mathrm{NO}_{5} \mathrm{Si}_{2} \mathrm{Na}(\mathrm{M}+\mathrm{Na})^{+}:$722.4607, found: 722.4607 ; calcd for $\mathrm{C}_{40} \mathrm{H}_{69} \mathrm{NO}_{5} \mathrm{Si}{ }_{2} \mathrm{~K}(\mathrm{M}+\mathrm{K})^{+}$: 738.4346, found: 738.4346 .

\section{(-)-\{(S)-1-[Hydroxy-bis-(4-tert-butyldimethylsilanyloxyphenyl)methyl]-2-methyl-}

propyl\}carbamic acid tert-butyl ester (2c). Compound 2c was prepared in an analogous fashion to the preparation of compound $\mathbf{2 b}$, using compound $\mathbf{1 c}(56.68 \mathrm{~g}, 197.3 \mathrm{mmol})$ and $\mathrm{N}$ (tert-butoxycarbonyl)- $L$-valine methyl ester $(13.05 \mathrm{~g}, 56.4 \mathrm{mmol})$ as starting materials. Purification via flash chromatography $\left(R_{\mathrm{f}}=0.25\right.$, 95:5 hexane / EtOAc) afforded compound 2c (33 g, $53.6 \mathrm{mmol}, 95 \%)$, as an amorphous solid. ${ }^{1} \mathrm{H}-\mathrm{NMR}\left(400 \mathrm{MHz}, \mathrm{CDCl}_{3}\right) \delta$ 7.37-7.26 (4H, m), 6.78-6.73 (4H, m), $5.07(1 \mathrm{H}, \mathrm{d}, J=12 \mathrm{~Hz}), 4.49(1 \mathrm{H}, \mathrm{m}), 2.74(1 \mathrm{H}, \mathrm{s}), 1.81(1 \mathrm{H}, \mathrm{m}), 1.45$ $(1 \mathrm{H}$, br. s), $1.35(9 \mathrm{H}, \mathrm{s}), 0.98(9 \mathrm{H}, \mathrm{s}), 0.97(9 \mathrm{H}, \mathrm{s}), 0.89(3 \mathrm{H}, \mathrm{d}, J=8 \mathrm{~Hz}), 0.85(3 \mathrm{H}, \mathrm{d}, J=4 \mathrm{~Hz})$, $0.19(6 \mathrm{H}, \mathrm{s}), 0.17(6 \mathrm{H}, \mathrm{s}) .{ }^{13} \mathrm{C}-\mathrm{NMR}\left(100 \mathrm{MHz}, \mathrm{CDCl}_{3}\right) \delta 160.8,158.7,158.6,143.9,143.0$, $133.8,131.5,131.0,125.7,124.5,124.0,123.9,86.3$, 83.4, 63.7, 33.1, 32.8, 30.1, 27.1, 22.6, 21.9, 0.1. Mass Spectrum (ESI, -MS) m/z 614 (M-H), 542 (M-tert-butanol). Specific Rotation 
$[\alpha]_{\mathrm{D}}{ }^{22^{\circ} \mathrm{C}}-31$ ( $c$ 1.0, DCM). HRMS $\left(\mathrm{ESI}^{+}\right)$calcd for $\mathrm{C}_{34} \mathrm{H}_{57} \mathrm{NO}_{5} \mathrm{Si}_{2} \mathrm{Na}(\mathrm{M}+\mathrm{Na})^{+}: 638.3668$, found: 638.3668; calcd for $\mathrm{C}_{34} \mathrm{H}_{57} \mathrm{NO}_{5} \mathrm{Si}_{2} \mathrm{~K}(\mathrm{M}+\mathrm{K})^{+}: 654.3407$, found: 654.3407 .

(-)-(S)-4-Isopropyl-5,5-bis-(4-triisopropylsilanyloxyphenyl)oxazolidin-2-one $\quad(3 b) . \quad$ The following protocol was adapted from a previously reported procedure. ${ }^{4}$ A solution of compound 2b (44 g, $62.8 \mathrm{mmol})$ in THF (300 mL) magnetically stirred on an ice bath under an atmosphere of nitrogen, was treated with potassium tert-butoxide $(8.9 \mathrm{~g}, 79.3 \mathrm{mmol})$ in one portion, and the reaction was allowed to warm slowly to RT over the course of $16 \mathrm{~h}$. The resultant mixture was treated with $\mathrm{NH}_{4} \mathrm{Cl}$ (300 $\mathrm{mL}$ of a $10 \% \mathrm{w} / \mathrm{v}$ aqueous solution) and stirred for $10 \mathrm{~min}$. The aqueous phase was separated and extracted with $\mathrm{Et}_{2} \mathrm{O}(2 \times 200 \mathrm{~mL})$ and the pooled organic phases were then dried $\left(\mathrm{MgSO}_{4}\right)$ and concentrated under reduced pressure. The resultant crude residue was purified via flash chromatography. Concentration of the appropriate fractions $\left(R_{\mathrm{f}}=0.3,4: 1\right.$ hexane / EtOAc) afforded compound $\mathbf{3 b}(33.5 \mathrm{~g}, 53.5 \mathrm{mmol}, 81 \%) .{ }^{1} \mathrm{H}-\mathrm{NMR}\left(400 \mathrm{MHz}, \mathrm{CDCl}_{3}\right)$ $\delta$ 7.33-7.31 (2H, m), 7.15-7.13 (2H, m), 6.87 (1H, br. s), 6.84-6.78 (4H, m), $4.23(1 \mathrm{H}, \mathrm{d}, J=4.0$ $\mathrm{Hz}), 1.76-1.73(1 \mathrm{H}, \mathrm{m}), 1.27-1.17(6 \mathrm{H}, \mathrm{m}), 1.09-1.06(36 \mathrm{H}$, complex m), $0.83(3 \mathrm{H}, \mathrm{d}, J=8.0$ $\mathrm{Hz}), 0.69(3 \mathrm{H}, \mathrm{d}, J=4.0 \mathrm{~Hz}) .{ }^{13} \mathrm{C}-\mathrm{NMR}\left(100 \mathrm{MHz}, \mathrm{CDCl}_{3}\right) \delta 159.4,156.1,155.7,136.5,132.4$, 128.2, 127.6, 119.8, 119.5, 89.6, 66.4, 29.9, 21.1, 18.1, 16.6, 12.9. Mass Spectrum (ESI, +MS) $m / z 626(\mathrm{M}+\mathrm{H})$. Specific Rotation $[\alpha]_{\mathrm{D}}{ }^{22^{\circ} \mathrm{C}}-122$ (c 1.0, MeOH). Anal. Calcd for $\mathrm{C}_{36} \mathrm{H}_{57} \mathrm{NO}_{4} \mathrm{Si}_{2}$ : C, 69.07; H, 9.50; N, 2.24\% Found: C, 68.96; H, 9.48; N, 2.16\%

\section{(-)-(S)-4-Isopropyl-5,5-bis-(4-tert-butyldimethylsilanyloxyphenyl)oxazolidin-2-one}

(3c).

Compound $\mathbf{3 c}$ was prepared in an analogous fashion to the preparation of compound $\mathbf{3 b}$, using compound 2c (26.8 g, $43.5 \mathrm{mmol})$ as starting material. Purification via flash chromatography $\left(R_{\mathrm{f}}\right.$ $=0.25,9: 1$ hexane $/$ EtOAc) afforded compound $3 \mathrm{c}(18.4 \mathrm{~g}, 33.9 \mathrm{mmol}, 78 \%$ ), as an amorphous solid. ${ }^{1} \mathrm{H}-\mathrm{NMR}\left(400 \mathrm{MHz}, \mathrm{CDCl}_{3}\right) \delta$ 7.36-7.33 (2H, m), 7.17-7.15 (2H, m), 6.81-6.74 (4H, m), $6.71(1 \mathrm{H}$, br. s), $4.23(1 \mathrm{H}, \mathrm{d}, \mathrm{J}=4.0 \mathrm{~Hz}), 1.80-1.73(1 \mathrm{H}, \mathrm{m}), 0.96(18 \mathrm{H}, \mathrm{m}), 0.83(3 \mathrm{H}, \mathrm{d}, \mathrm{J}=8.0$ $\mathrm{Hz}), 0.70(3 \mathrm{H}, \mathrm{d}, \mathrm{J}=4.0 \mathrm{~Hz}), 0.18(12 \mathrm{H}, \mathrm{m})$.

${ }^{13} \mathrm{C}-\mathrm{NMR}\left(100 \mathrm{MHz}, \mathrm{CDCl}_{3}\right) \delta 163.5,159.8,159.5,141.0,136.6,132.3,131.7,124.1,123.8$, 93.8, 70.6, 34.1, 30.0, 25.2, 22.6, 20.6, 0.01. Mass Spectrum (ESI, +MS) m/z $542(\mathrm{M}+\mathrm{H})$. Specific Rotation $[\alpha]_{\mathrm{D}}{ }^{2{ }^{\circ} \mathrm{C}}-137(c$ 1.0, $\mathrm{MeOH})$. Anal. Calcd for $\mathrm{C}_{30} \mathrm{H}_{47} \mathrm{NO}_{4} \mathrm{Si}_{2}$ : C, 66.50; H, 8.74; N, 2.58\% Found: C, 66.21; H, 8.73; N, 2.52\%

(-)-(S)-5,5-Bis-(4-hydroxyphenyl)-4-isopropyloxazolidin-2-one (3a). A dry polyethylene bottle flushed with nitrogen was charged with a magnetic stirrer and a solution of compound $\mathbf{3 b}$ (17.5 g, $27.9 \mathrm{mmol})$ in THF (150 mL) and cooled in an ice bath with stirring, while HF.pyridine (35 $\mathrm{mL}$ of a $65-70 \%$ commercial solution) was added dropwise. The reaction was allowed to warm to RT over $16 \mathrm{~h}$. The resultant mixture was diluted with diethyl ether $(100 \mathrm{~mL})$ and sat. aq. $\mathrm{NaHCO}_{3}(500 \mathrm{~mL})$. Solid $\mathrm{NaHCO}_{3}$ was then added, in portions, until the reaction mixture was neutralised as evidenced by no more $\mathrm{CO}_{2}$ being liberated. The organic layer was separated and the aqueous layer was extracted with 50:50 THF / diethyl ether ( 2 × $200 \mathrm{~mL})$. The pooled organic layers were washed with brine, dried $\left(\mathrm{MgSO}_{4}\right)$, and concentrated under reduced pressure. The residue was purified via flash chromatography. Concentration of the appropriate fractions $\left(R_{\mathrm{f}}=0.45,1: 9 \mathrm{MeOH} / \mathrm{DCM}\right)$ afforded compound 3a $(7.36 \mathrm{~g}, 23.5 \mathrm{mmol}, 84 \%)$. This protocol 
was also used to prepare compound 3a, in similar yield, via deprotection of compound $\mathbf{3 c} .{ }^{1} \mathrm{H}$ NMR (400 MHz, CD 3 OD) $\delta$ 7.35-7.33 (2H, m), 7.16-7.13 (2H, m), 6.79-6.73 (4H, m), $4.96(2 \mathrm{H}$, br. s), $4.32(1 \mathrm{H}, \mathrm{m}), 1.85-1.73(1 \mathrm{H}, \mathrm{m}), 0.86(3 \mathrm{H}, \mathrm{d}, J=8.0 \mathrm{~Hz}), 0.64(3 \mathrm{H}, \mathrm{d}, J=8.0 \mathrm{~Hz}) .{ }^{13} \mathrm{C}-$ NMR (100 MHz, $\left.\mathrm{CD}_{3} \mathrm{OD}\right) \delta 160.0,157.1,156.7,135.7,130.9,127.6,127.2,114.9,114.6,89.9$, 67.7, 65.7, 29.8, 25.4, 20.0, 15.0. Mass Spectrum (ESI, +MS) m/z $314(\mathrm{M}+\mathrm{H}), 270\left(\mathrm{M}-\mathrm{CO}_{2}\right)$. Specific Rotation $[\alpha]_{\mathrm{D}}{ }^{22^{\circ} \mathrm{C}}-218\left(c\right.$ 1.0, MeOH). Anal. Calcd for $\mathrm{C}_{18} \mathrm{H}_{19} \mathrm{NO}_{4}$ : C, 68.99; H, 6.11; N, 4.47\% Found: C, 67.80; H, 7.08; N, 3.95\%

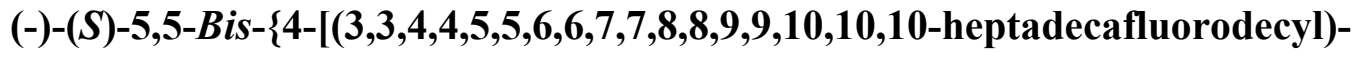

diisopropylsilanyloxy]phenyl\}-4-isopropyloxazolidin-2-one (3d). The following protocol was adapted from a previously reported procedure. ${ }^{10}$ A $50 \mathrm{~mL}$ round-bottomed flask fitted with a septum and nitrogen entry and exit needles was charged with commercially available $\left(1 \mathrm{H}, 1 \mathrm{H}, 2 \mathrm{H}, 2 \mathrm{H}\right.$-perfluorodecyl)diisopropylsilane $(4.84 \mathrm{~g}, 8.61 \mathrm{mmol})$ and then cooled to $-10{ }^{\circ} \mathrm{C}$ on a dry-ice / acetone bath. Tert-butyl hypochlorite $(0.851 \mathrm{~g}, 7.84 \mathrm{mmol})$ was then added, dropwise, via syringe. ${ }^{12}$ After 5 minutes, the reaction vessel was placed on a rotary evaporator and heated to $70^{\circ} \mathrm{C}$ under a reduced pressure of $\sim 30 \mathrm{mBar}$ to remove tert-butanol. To this vessel was then added a solution of compound $\mathbf{3 a}(1.22 \mathrm{~g}, 3.89 \mathrm{mmol})$ in THF (10 mL) with stirring, at RT, under an atmosphere of nitrogen. Imidazole (1.06 g, $15.57 \mathrm{mmol})$ was then added, in a single portion, and stirring was continued at this temperature for $65 \mathrm{~h}$. The mixture was diluted with diethyl ether $(50 \mathrm{~mL})$ and washed sequentially with $\mathrm{HCl}(2 \times 20 \mathrm{~mL}$ of a $0.5 \mathrm{M}$ aqueous solution), sat. aq. $\mathrm{NaHCO}_{3}(20 \mathrm{~mL})$ and $\mathrm{H}_{2} \mathrm{O}(20 \mathrm{~mL})$, then dried $\left(\mathrm{MgSO}_{4}\right)$ and concentrated under reduced pressure. The resulting crude oil was purified via flash chromatography. Concentration of the appropriate fractions $\left(R_{\mathrm{f}}=0.4,1: 4\right.$ EtOAc / hexane) gave compound 3d (3.2 g, $2.28 \mathrm{mmol}, 59 \%)$. ${ }^{1} \mathrm{H}-\mathrm{NMR}\left(400 \mathrm{MHz}, \mathrm{CDCl}_{3}\right) \delta 7.35(2 \mathrm{H}, \mathrm{m}), 7.18(2 \mathrm{H}, \mathrm{m}), 6.79(4 \mathrm{H}$, m), $4.25(1 \mathrm{H}, \mathrm{d}, J=4.0 \mathrm{~Hz}), 2.20-2.00(4 \mathrm{H}, \mathrm{m}), 1.78-1.68(1 \mathrm{H}, \mathrm{m}), 1.24-1.13(4 \mathrm{H}$, complex m), 1.09-1.02 (24H, m), 1.01-0.95 (4H, m), $0.84(3 \mathrm{H}, \mathrm{d}, J=8.0 \mathrm{~Hz}), 0.70(3 \mathrm{H}, \mathrm{d}, J=4.0 \mathrm{~Hz}) .{ }^{13} \mathrm{C}-$ NMR $\left(100 \mathrm{MHz}, \mathrm{CDCl}_{3}\right) \delta 159.3,155.3,154.9,137.1,133.0,131.1,128.4,127.8,119.6,119.2$, $110.0,89.4,66.2,29.9,20.9,17.51,17.49,17.46,17.41,17.39,17.37,16.3,13.0 .{ }^{19} \mathrm{~F}-\mathrm{NMR}(376$ $\left.\mathrm{MHz}, \mathrm{CDCl}_{3}\right) \delta$-81.35--81.40 (6F, m), -117.06--117.23 (4F, m), -122.32 (4F, s), -122.49 (8F, s), -123.29 (4F, s), -123.80 (4F, s), -126.71 (4F, s). Mass Spectrum (ESI, +MS) m/z 1435 (M+H). Specific Rotation $[\alpha]_{\mathrm{D}}{ }^{2{ }^{\circ} \mathrm{C}}-51(c 1.0, \mathrm{MeOH})$.

\section{(-)-(S)-5,5-Bis-[4-(2,2,3,3,4,4,5,5,6,6,7,7,8,8,9,9-hexadecafluorononyloxy)-phenyl]-4-}

isopropyloxazolidin-2-one (3e). The following protocol was adapted from a previously reported procedure. ${ }^{11}$ A mixture of compound 3a (6.75 g, $\left.21.56 \mathrm{mmol}\right), 2,2,3,3,4,4,5,5,6,6,7,7,8,8,9,9$ Hexadecafluoro-nonan-1-trifluoromethanesulfonate $(29.58 \mathrm{~g}, 52.43 \mathrm{mmol})$ prepared as previously described ${ }^{12}$ and $\mathrm{Cs}_{2} \mathrm{CO}_{3}(21.5 \mathrm{~g}, 65.9 \mathrm{mmol})$ in DMF $(140 \mathrm{ml})$ was stirred, under a nitrogen atmosphere, at a temperature of $120^{\circ} \mathrm{C}$, for $72 \mathrm{~h}$. The resultant suspension was cooled to $\mathrm{RT}$, then placed on an ice bath, and treated with $\mathrm{HCl}(250 \mathrm{~mL}$ of a $0.5 \mathrm{M}$ aqueous solution). After stirring for $5 \mathrm{~min}$, the mixture was diluted with diethyl ether $(500 \mathrm{~mL})$ and poured into a separation funnel. The aqueous phase was separated, and the organic phase was washed with $\mathrm{HCl}\left(2 \times 250 \mathrm{~mL}\right.$ of a $0.5 \mathrm{M}$ aqueous solution), then dried $\left(\mathrm{MgSO}_{4}\right)$ and concentrated under 
reduced pressure. The residue was purified via flash chromatography. Concentration of the appropriate fractions $\left(R_{\mathrm{f}}=0.2\right.$, DCM elution) afforded compound $3 \mathrm{e}(10.58 \mathrm{~g}, 9.27 \mathrm{mmol}, 43 \%)$. ${ }^{1} \mathrm{H}-\mathrm{NMR}\left(400 \mathrm{MHz}, \mathrm{CDCl}_{3}\right)$ $\delta$ 7.47-7.45 (2H, m), 7.31-7.29 (2H, m), 6.93-6.88 (4H, m), 6.19$5.91(2 \mathrm{H}, \mathrm{tt}, J=4.0$ and $52.0 \mathrm{~Hz}), 6.10(1 \mathrm{H}$, br. s), $4.44(4 \mathrm{H}, \mathrm{t}, J=12.0 \mathrm{~Hz}), 4.27(1 \mathrm{H}, \mathrm{d}, J=4.0$ $\mathrm{Hz}), 1.80-1.75(1 \mathrm{H}, \mathrm{m}), 0.87(3 \mathrm{H}, \mathrm{d}, J=8.0 \mathrm{~Hz}), 0.70(3 \mathrm{H}, \mathrm{d}, J=8.0 \mathrm{~Hz}) .{ }^{13} \mathrm{C}-\mathrm{NMR}(100 \mathrm{MHz}$, $\left.\mathrm{CDCl}_{3}\right) \delta 159.3,157.4,157.0,138.2,133.6,128.1,127.6,155.0,114.6,107.7,89.1,66.1,66.0$, 65.6, 65.4, 65.3, 29.9, 20.9, 15.9. ${ }^{19} \mathrm{~F}-\mathrm{NMR}\left(376 \mathrm{MHz}, \mathrm{CDCl}_{3}\right) \delta-120.1$ (4F, s), -122.5 (12F, s), 123.7 (4F, s), -123.9 (4F, s), -130.0 (4F, s), -137.7 (4F, dt, $J=45.1$ and 7.5 Hz). Mass Spectrum $(\mathrm{ESI},+\mathrm{MS}) m / z 1142(\mathrm{M}+\mathrm{H})$. Specific Rotation $[\alpha]_{\mathrm{D}}{ }^{22^{\circ} \mathrm{C}}-62\left(c\right.$ 1.0, MeOH). HRMS (ESI $\left.{ }^{+}\right)$calcd for $\mathrm{C}_{36} \mathrm{H}_{24} \mathrm{~F}_{32} \mathrm{NO}_{4}(\mathrm{M}+\mathrm{H})^{+}:$: 1142.1189, found: 1142.1189 ; calcd for $\mathrm{C}_{36} \mathrm{H}_{23} \mathrm{~F}_{32} \mathrm{NO}_{4} \mathrm{Na}(\mathrm{M}+\mathrm{Na})^{+}$: 1164.1008, found: 1164.1015 .

\section{[(S)-1-\{Hydroxy-bis-[4-(3,3,4,4,5,5,6,6,7,7,8,8,8-tridecafluorooctyl)phenyl]methyl\}-2-}

methylpropyl]carbamic acid tert-butyl ester (5). A magnetically stirred solution of commercially available 1-bromo-4-(3,3,4,4,5,5,6,6,7,7,8,8,8-tridecafluorooctyl)benzene (4, $5 \mathrm{~g}$, $9.9 \mathrm{mmol})$ in THF $(10 \mathrm{~mL})$ maintained at $-78{ }^{\circ} \mathrm{C}$ under an atmosphere of nitrogen was treated, dropwise, with $n$-BuLi $(6.2 \mathrm{~mL}$ of a $1.6 \mathrm{M}$ solution in hexane, $9.92 \mathrm{mmol})$ and stirring was continued at this temperature for 20 minutes. The suspension was allowed to warm to $-50{ }^{\circ} \mathrm{C}$ for 10 minutes and then cooled again to $-78{ }^{\circ} \mathrm{C}$, before being treated, dropwise, with $N$-(tertbutoxycarbonyl)- $L$-valine methyl ester $(0.93 \mathrm{~g}, 4.0 \mathrm{mmol})$ in THF $(5 \mathrm{~mL})$. Stirring was continued at $-78{ }^{\circ} \mathrm{C}$ for $6 \mathrm{~h}$ before quenching, at this temperature, with sat. aq. $\mathrm{NH}_{4} \mathrm{Cl}(25 \mathrm{~mL})$. The resultant suspension was diluted with diethyl ether $(50 \mathrm{~mL})$. The aqueous phase was separated and the organic phase was washed sequentially with sat. aq. $\mathrm{NH}_{4} \mathrm{Cl}(40 \mathrm{~mL})$ and brine $(40 \mathrm{~mL})$, then dried $\left(\mathrm{MgSO}_{4}\right)$ and concentrated under reduced pressure. The residue was purified via flash chromatography. Concentration of the appropriate fractions $\left(R_{\mathrm{f}}=0.35,9: 1\right.$ hexane / EtOAc) yielded the desired compound 5 (3.4 g, $3.25 \mathrm{mmol}, \sim 82 \%)$, albeit impure due to coeluting $N$-(tert-butoxycarbonyl)- $L$-valine methyl ester starting material. This material was not characterised but rather was employed immediately in the next step.

(-)-(S)-4-Isopropyl-5,5-bis-[4-(3,3,4,4,5,5,6,6,7,7,8,8,8-tridecafluorooctyl)phenyl]-oxazolidin2-one (6). The following protocol was adapted from a previously reported procedure. ${ }^{4} \mathrm{~A}$ magnetically stirred solution of compound $5(3.4 \mathrm{~g}, 3.25 \mathrm{mmol})$ maintained at $0{ }^{\circ} \mathrm{C}$ under an atmosphere of nitrogen, was treated with potassium tert-butoxide $(0.44 \mathrm{~g}, 3.9 \mathrm{mmol})$ in one portion, and the resultant mixture was allowed to slowly warm to RT over the course of $16 \mathrm{~h}$. The suspension was treated with $\mathrm{NH}_{4} \mathrm{Cl}(10 \mathrm{~mL}$ of a $10 \%$ aqueous solution). The aqueous phase was separated and washed with diethyl ether $(2 \times 20 \mathrm{~mL})$. The pooled organic phases were then dried and concentrated under reduced pressure. The resultant crude material was then purified via flash chromatography. Concentration of the appropriate fractions $\left(R_{\mathrm{f}}=0.3,3: 1\right.$ hexane / EtOAc) afforded compound 6 (1.8 g, $1.85 \mathrm{mmol}, 57 \%) .{ }^{1} \mathrm{H}-\mathrm{NMR}\left(400 \mathrm{MHz}, \mathrm{CDCl}_{3}\right) \delta 7.49(2 \mathrm{H}$, m), $7.34(2 \mathrm{H}, \mathrm{m}), 7.17(4 \mathrm{H}, \mathrm{m}), 7.15(1 \mathrm{H}$, br. s), $4.33(1 \mathrm{H}, \mathrm{d}, J=4.0 \mathrm{~Hz}), 2.91-2.84(4 \mathrm{H}$, complex m), 2.41-2.25 (4H, complex m), 1.87-1.81 (1H, complex m), $0.92(3 \mathrm{H}, \mathrm{d}, J=8.0 \mathrm{~Hz})$, $0.70(3 \mathrm{H}, \mathrm{d}, J=4.0 \mathrm{~Hz}) .{ }^{13} \mathrm{C}-\mathrm{NMR}\left(100 \mathrm{MHz}, \mathrm{CDCl}_{3}\right) \delta 159.5,142.8,139.4,138.8,137.9$, 
$128.7,128.2,126.8,126.2,89.3,66.2,53.6,33.2,33.1,32.9,32.8,32.7,32.6,29.9,26.1,20.9$, 15.7 .

${ }^{19} \mathrm{~F}-\mathrm{NMR}\left(376 \mathrm{MHz}, \mathrm{CDCl}_{3}\right) \delta-81.35(6 \mathrm{~F}, \mathrm{~m}),-115.20(4 \mathrm{~F}, \mathrm{~m}),-122.41(4 \mathrm{~F}, \mathrm{~m}),-123.41(4 \mathrm{~F}, \mathrm{~s})$, -124.05 (4F, m), -126.70 (4F, m). Mass Spectrum (ESI, +MS) m/z 974 (M+H). Specific Rotation $[\alpha]_{\mathrm{D}}{ }^{22^{\circ} \mathrm{C}}-79(c 1.0, \mathrm{MeOH})$. HRMS $\left(\mathrm{ESI}^{+}\right)$calcd for $\mathrm{C}_{34} \mathrm{H}_{26} \mathrm{~F}_{26} \mathrm{NO}_{2}(\mathrm{M}+\mathrm{H})^{+}: 974.1543$, found: 974.1550; calcd for $\mathrm{C}_{34} \mathrm{H}_{29} \mathrm{~F}_{36} \mathrm{~N}_{2} \mathrm{O}_{2}\left(\mathrm{M}+\mathrm{NH}_{4}\right)^{+}$: 991.1808, found: 991.1813; $\mathrm{C}_{34} \mathrm{H}_{25} \mathrm{~F}_{36} \mathrm{NO}_{2} \mathrm{Na}$ $(\mathrm{M}+\mathrm{Na})^{+}$: 996.1362, found: 996.1366; $\mathrm{C}_{34} \mathrm{H}_{25} \mathrm{~F}_{36} \mathrm{NO}_{2} \mathrm{~K}(\mathrm{M}+\mathrm{K})^{+}: 1012.1102$, found: 1012.1102 .

\section{(-)-(S)-4-Isopropyl-5,5-bis-[4-(3,3,4,4,5,5,6,6,7,7,8,8,8-tridecafluorooctyl)phenyl]-oxazolidin-} 2-one (6): One-pot procedure

To a magnetically stirred solution of 1-bromo-4-(3,3,4,4,5,5,6,6,7,7,8,8,8-tridecafluorooctyl)benzene $(4,40.83 \mathrm{~g}, 81.1 \mathrm{mmol})$ in THF $(400 \mathrm{~mL})$ maintained at $-78{ }^{\circ} \mathrm{C}$ under an atmosphere of nitrogen, was added $n-\mathrm{BuLi}(50.63 \mathrm{~mL}$ of a $1.6 \mathrm{M}$ solution in hexane, $81.0 \mathrm{mmol})$ and stirring was continued at this temperature for $20 \mathrm{~min}$. The resultant suspension was then treated, dropwise, with $N$-(tert-butoxycarbonyl)- $L$-valine methyl ester $(7.5 \mathrm{~g}, 32.4 \mathrm{mmol})$ in THF $(10 \mathrm{~mL})$ and stirring was continued at $-78^{\circ} \mathrm{C}$ for $6 \mathrm{~h}$. After this time, the dry ice-actone bath was removed and the mixture was allowed to warm to RT with continued stirring for $16 \mathrm{~h}$. The reaction was then quenched with sat. aq. $\mathrm{NH}_{4} \mathrm{Cl}(200 \mathrm{~mL})$ and diluted with diethyl ether $(400 \mathrm{~mL})$. The aqueous layer was separated and the organic layer was washed sequentially with sat. aq. $\mathrm{NH}_{4} \mathrm{Cl}(200 \mathrm{~mL})$ and brine $(200 \mathrm{~mL})$, then dried $\left(\mathrm{MgSO}_{4}\right)$ and concentrated under reduced pressure. The residue was purified via flash chromatography. Concentration of the appropriate fractions $\left(R_{\mathrm{f}}=0.3,3: 1\right.$ hexane / EtOAc) afforded compound $6(15.3 \mathrm{~g}, 15.72 \mathrm{mmol}$, $50 \%$ over two steps). This material was identical, as judged by ${ }^{1} \mathrm{H}-\mathrm{NMR},{ }^{13} \mathrm{C}-\mathrm{NMR},{ }^{19} \mathrm{~F}-\mathrm{NMR}$ and low resolution mass spectrometric analysis, as well as chromatographic behaviour (TLC and flash-column chromatography), with the material prepared via the two-step process reported above.

\section{$(+)-(R)$-4-Isopropyl-5,5-bis-[4-(3,3,4,4,5,5,6,6,7,7,8,8,8-tridecafluorooctyl)phenyl]-}

oxazolidin-2-one (ent-6). Compound ent-6 was prepared in an analogous fashion to the preparation of compound 6 (one-pot procedure), using compound $N$-(tert-butoxycarbonyl)- $D$ valine methyl ester $(5.1 \mathrm{~g}, 22 \mathrm{mmol})$ as starting material. Purification via flash chromatography $\left(R_{\mathrm{f}}=0.3,3: 1\right.$ hexane / EtOAc) afforded compound ent-6 (10.04 g, 10.3 mmol, 47\% over two steps). This material was identical, as judged by ${ }^{1} \mathrm{H}-\mathrm{NMR},{ }^{13} \mathrm{C}-\mathrm{NMR},{ }^{19} \mathrm{~F}-\mathrm{NMR}$ and low resolution mass spectrometric analysis, as well as chromatographic behaviour (TLC and flashcolumn chromatography), with the optical antipode compound $\mathbf{6}$ reported above.

Specific Rotation $[\alpha]_{\mathrm{D}}^{22^{\circ} \mathrm{C}}+78(c 1.0, \mathrm{MeOH})$.

\section{(-)-(S)-3-(2-Benzylacryloyl)-4-isopropyl-5,5-bis-[4-(3,3,4,4,5,5,6,6,7,7,8,8,8-}

tridecafluorooctyl)phenyl]oxazolidin-2-one (7). The following protocol was adapted from a previously reported procedure. ${ }^{1}$ A magnetically stirred suspension of compound 6 (3.88 g, $3.99 \mathrm{mmol})$, DMAP $(0.098 \mathrm{~g}, 0.8 \mathrm{mmol})$ and 2-benzyl-acrylic acid $(1.3 \mathrm{~g}, 8.0 \mathrm{mmol})$ in DCM $(20 \mathrm{~mL})$, maintained at $0{ }^{\circ} \mathrm{C}$ under an atmosphere of nitrogen, was treated with DIPCD $(1.24 \mathrm{~mL}$, 
$1.01 \mathrm{~g}, 8.0 \mathrm{mmol}$ ). After $10 \mathrm{~min}$, the reaction was allowed to warm to RT and stirring was continued for $16 \mathrm{~h}$. The diisopropyl urea formed was filtered, and the precipitate washed with DCM $(10 \mathrm{~mL})$. The filtrate was washed with sat. aq. $\mathrm{NaHCO}_{3}(10 \mathrm{~mL})$, then dried $\left(\mathrm{MgSO}_{4}\right)$ and concentrated under reduced pressure. The residue was purified via flash chromatography. Concentration of the appropriate fractions $\left(R_{\mathrm{f}}=0.3,95: 5\right.$ hexane / EtOAc) gave compound 7 (3.5 g, $3.13 \mathrm{mmol}, 78 \%)$. ${ }^{1} \mathrm{H}-\mathrm{NMR}\left(400 \mathrm{MHz}, \mathrm{CDCl}_{3}\right) \delta 7.41(2 \mathrm{H}, \mathrm{m}), 7.36(2 \mathrm{H}, \mathrm{m}), 7.26-7.10$ $(9 \mathrm{H}$, complex m), $5.35(1 \mathrm{H}, \mathrm{d}, J=4.0 \mathrm{~Hz}), 5.20(2 \mathrm{H}, \mathrm{s}), 3.65(2 \mathrm{H}, \mathrm{s}), 2.92-2.85(4 \mathrm{H}$, complex m), 2.40-2.20 (4H, complex m), 2.00-1.90 (1H, m), $0.81(3 \mathrm{H}, \mathrm{d}, J=4.0 \mathrm{~Hz}), 0.73(3 \mathrm{H}, \mathrm{d}, J=8.0$ $\mathrm{Hz}) .{ }^{13} \mathrm{C}-\mathrm{NMR}\left(100 \mathrm{MHz}, \mathrm{CDCl}_{3}\right) \delta 170.2,152.3,143.2,141.1,139.8,139.2,137.3,136.8$, 129.5, 129.0, 128.6, 126.7, 126.5, 126.2, 120.5, 89.3, 68.2, 64.9, 39.5, 32.8, 30.2, 28.1, 26.2, 26.1, 25.8, 21.7, 16.2. ${ }^{19} \mathrm{~F}-\mathrm{NMR}\left(376 \mathrm{MHz}, \mathrm{CDCl}_{3}\right) \delta-81.33(6 \mathrm{~F}, \mathrm{~m}),-115.19(4 \mathrm{~F}, \mathrm{~m}),-122.41$ (4F, s), -123.39 (4F, s), -124.01 (4F, s), -126.69 (4F, s). Mass Spectrum (ESI, +MS) m/z 1118 $(\mathrm{M}+\mathrm{H})$. Specific Rotation $[\alpha]_{\mathrm{D}}{ }^{2{ }^{\circ} \mathrm{C}}-42(c 1.0, \mathrm{MeOH})$.

(+)-(R)-3-(2-Benzyl-acryloyl)-4-isopropyl-5,5-bis-[4-(3,3,4,4,5,5,6,6,7,7,8,8,8tridecafluorooctyl)phenyl]oxazolidin-2-one (ent-7). Compound ent-7 was prepared in an analogous fashion to the preparation of compound 7, using compound ent-6 (6.94 g, $7.1 \mathrm{mmol})$ as starting material. Purification via flash chromatography $\left(R_{\mathrm{f}}=0.3,95: 5\right.$ hexane / EtOAc) afforded compound ent-7 (4.3 g, $3.85 \mathrm{mmol}, 54 \%)$. This material was identical, as judged by ${ }^{1} \mathrm{H}-$ NMR, ${ }^{13} \mathrm{C}-\mathrm{NMR},{ }^{19} \mathrm{~F}-\mathrm{NMR}$ and low resolution mass spectrometric analysis, as well as chromatographic behaviour (TLC and flash-column chromatography), with the optical antipode compound 6 reported above. Specific Rotation $[\alpha]_{\mathrm{D}}{ }^{2{ }^{\circ} \mathrm{C}}+46(c 1.0, \mathrm{MeOH})$.

(S)-3-[(R)-2-(Benzyloxyamino-methyl)-3-phenylpropionyl]-4-isopropyl-5,5-bis-[4-

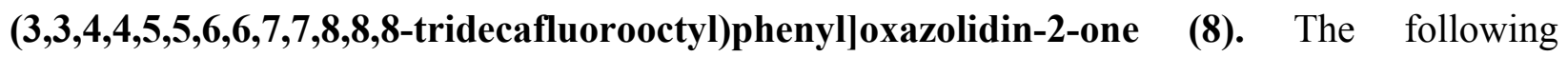
protocol was adapted from a previously reported procedure. ${ }^{6}$ A dry round-bottomed flask was charged with a magnetic stirrer bead, compound $7(3.11 \mathrm{~g}, 2.78 \mathrm{mmol}), O$-benzyl hydroxylamine $(0.684 \mathrm{~g}, 5.55 \mathrm{mmol})$ and THF $(10 \mathrm{~mL})$. The resultant solution was heated, with stirring, to $72{ }^{\circ} \mathrm{C}$ under an atmosphere of nitrogen. The reaction was allowed to proceed at this temperature for $24 \mathrm{~h}$. The mixture was then cooled to RT, concentrated under reduced pressure and purified via FSPE. A standard glass flash chromatography column was packed with commercially available Fluoroflash ${ }^{\mathrm{TM}}(35 \mathrm{~g})$ and pre-conditioned by passing through $\mathrm{MeOH} / \mathrm{H}_{2} \mathrm{O}(80 \mathrm{~mL}$ of an $80: 20 \mathrm{v} / \mathrm{v}$ mixture) under positive pressure. The residue was loaded on to the top of the column, using DMF (4 mL) as the loading solvent. The column was then eluted with $\mathrm{MeOH}$ / $\mathrm{H}_{2} \mathrm{O}(80 \mathrm{~mL}$ of an $80: 20 \mathrm{v} / \mathrm{v}$ mixture $)$. Concentration of this fraction under reduced pressure afforded $o$-benzyl hydroxylamine $(0.27 \mathrm{~g}, 2.13 \mathrm{mmol})$. The column was then eluted successively with $\mathrm{MeOH}(100 \mathrm{~mL})$, THF $(120 \mathrm{~mL})$ and acetone $(120 \mathrm{~mL})$. The three organic fractions were pooled and concentrated under reduced pressure to afford a clear colourless oil containing the desired compound 8 (3.24 g, $2.61 \mathrm{mmol}$, 94\%). Subjection of this material to chiral HPLC analysis (Chiralpak ${ }^{\mathrm{TM}}$ AD-H 1196 analytical column, 2:98 v/v isopropanol-hexane elution, flowrate $1.0 \mathrm{~mL} / \mathrm{min}$ ) confirmed that it had been obtained in $80 \%$ d.e: $R_{\mathrm{t}} 12.85$ (major diastereomer), $16.96 \mathrm{~min}$ (minor diastereomer). A magnetically stirred solution of compound $\mathbf{8}$ 
(3.18 g, $2.56 \mathrm{mmol})$, in diethyl ether $(250 \mathrm{~mL})$ maintained at RT under an atmosphere of nitrogen, was treated with a solution of $p$-toluenesulfonic acid monohydrate $(512 \mathrm{mg}$, $2.73 \mathrm{mmol})$ in diethyl ether $(20 \mathrm{~mL})$ and stirring was continued for $72 \mathrm{~h}$. After this time, the resultant suspension was cooled to $0{ }^{\circ} \mathrm{C}$ on an ice bath, and then filtered. The solid material was washed with ice cold diethyl ether $(2 \times 25 \mathrm{~mL})$ and dried under reduced pressure to afford the toluene sulfonic acid salt of compound 8 (2.53 g, $1.79 \mathrm{mmol}, 70 \%, 93 \%$ d.e. by $\left.{ }^{1} \mathrm{H}-\mathrm{NMR}\right)$. This material was suspended in EtOAc $(20 \mathrm{~mL})$ and treated with sodium carbonate $(10 \mathrm{~mL}$ of a $1 \mathrm{M}$ aqueous solution), whilst being magnetically stirred at r.t. under an atmosphere of nitrogen. After $15 \mathrm{~min}$, the aqueous layer was separated and discarded. The organic layer was washed with water $(2 \times 10 \mathrm{~mL})$, then dried $\left(\mathrm{MgSO}_{4}\right)$, and concentrated under reduced pressure to afford compound 8 (2.06 g, $1.66 \mathrm{mmol}, 93 \%)$. Subjection of this material to chiral HPLC analysis (Chiralpak ${ }^{\mathrm{TM}}$ AD-H 1196 analytical column, 2:98 v/v isopropanol-hexane elution, flow rate $1.0 \mathrm{~mL} / \mathrm{min}$ ) confirmed that it had been obtained in $93 \%$ d.e: $R_{\mathrm{t}} 12.85$ (major diastereomer), $16.96 \mathrm{~min}$ (minor diastereomer). ${ }^{1} \mathrm{H}-\mathrm{NMR}\left(400 \mathrm{MHz}, \mathrm{CDCl}_{3}\right) \delta$ 7.35-6.94 (18H, complex m), 5.74 (1H, br. s), $5.35(1 \mathrm{H}, \mathrm{d}, J=4.0 \mathrm{~Hz}), 4.66(2 \mathrm{H}, \mathrm{m}), 4.35-4.29$ (1H, complex m), 3.31-3.26 $(1 \mathrm{H}$, complex m), 3.10-3.06 $(1 \mathrm{H}$, complex $\mathrm{m}), 2.92-2.83(4 \mathrm{H}$, complex m), 2.73-2.66 $(1 \mathrm{H}$, complex m), 2.56-2.51 (1H, complex m), 2.42-2.21 (4H, complex m), 1.97-1.90 (1H, complex m), $0.91(3 \mathrm{H}, \mathrm{d}, J=8.0 \mathrm{~Hz}), 0.76(3 \mathrm{H}, \mathrm{d}, J=8.0 \mathrm{~Hz})$. Mass Spectrum (ESI, $+\mathrm{MS}) \mathrm{m} / z 1241$ $(\mathrm{M}+\mathrm{H})$. Anal. Calcd for $\mathrm{C}_{58} \mathrm{H}_{50} \mathrm{~F}_{26} \mathrm{~N}_{2} \mathrm{O}_{7} \mathrm{~S}: \mathrm{C}, 49.30 ; \mathrm{H}, 3.57 ; \mathrm{N}, 1.98 \%$ Found: C, 49.14; H, 3.54; $\mathrm{N}, 1.94 \%$

\section{(R)-3-[(S)-2-(Benzyloxyaminomethyl)-3-phenylpropionyl]-4-isopropyl-5,5-bis-[4-}

$(3,3,4,4,5,5,6,6,7,7,8,8,8$-tridecafluorooctyl)phenyl]oxazolidin-2-one (ent-8). Compound ent-8 was prepared using the same protocols as those described for the preparation of compound $\mathbf{8}$, using compound ent-7 (3.5 g, $3.13 \mathrm{mmol})$ as starting material. Purification via the same means (FSPE \& precipitation of tosylate) afforded compound ent-8 (2.25 g, $1.6 \mathrm{mmol}, 51 \%$ over the two steps). This material was identical, as judged by ${ }^{1} \mathrm{H}-\mathrm{NMR},{ }^{13} \mathrm{C}-\mathrm{NMR},{ }^{19} \mathrm{~F}-\mathrm{NMR}$ and low resolution mass spectrometric analysis, as well as chromatographic behaviour (TLC and flashcolumn chromatography), with the optical antipode compound 8 reported above.

(R)-2-(Benzyloxyaminomethyl)-3-phenylpropionic acid (9) and recovery of compound 6 via FSPE. A magnetically stirred solution of compound 8 (2.06 g, $1.66 \mathrm{mmol}, 93 \%$ d.e $)$, in THF $(80 \mathrm{~mL})$ and water $(10 \mathrm{~mL})$, maintained at $0{ }^{\circ} \mathrm{C}$ under an atmosphere of nitrogen, was treated with $\mathrm{H}_{2} \mathrm{O}_{2}$ (40 mL of a $30 \%$ aqueous solution), followed by $\mathrm{LiOH}$ (160 mL of a $4.5 \%$ aqueous solution). The reaction mixture was stirred at this temperature for $45 \mathrm{~min}$, before being quenched via addition of cold sat. aq. sodium sulfite $(40 \mathrm{~mL})$, and allowed to warm to RT over the course of $30 \mathrm{~min}$. The resultant suspension was extracted with EtOAc (4 x $100 \mathrm{~mL})$ and the organic extracts dried $\left(\mathrm{MgSO}_{4}\right)$, and concentrated under reduced pressure to give a residue which was subjected to FSPE. A commercially available Fluoroflash ${ }^{\mathrm{TM}}$ cartridge $(20 \mathrm{~g})$ was preconditioned by passing through $\mathrm{MeOH} / \mathrm{H}_{2} \mathrm{O}(60 \mathrm{~mL}$ of an $80: 20 \mathrm{v} / \mathrm{v}$ mixture $)$ under positive pressure. The residue was loaded on to the top of the column, using DMF (4 mL) as the loading solvent. The column was then eluted with $\mathrm{MeOH} / \mathrm{H}_{2} \mathrm{O}(60 \mathrm{~mL}$ of an $80: 20 \mathrm{v} / \mathrm{v}$ mixture). 
Concentration of this fraction under reduced pressure afforded non-fluorinated organic impurities. The column was then eluted successively with $\mathrm{MeOH}(80 \mathrm{~mL})$, THF (100 mL) and acetone $(100 \mathrm{~mL})$. The three organic fractions were pooled and concentrated under reduced pressure to afford recovered compound 6 (1.56 g, $1.60 \mathrm{mmol}, 96 \%)$ of high purity, as determined by ${ }^{1} \mathrm{H}-\mathrm{NMR}$ and LCMS. The aqueous layer from the original solvent extraction was acidified with conc. $\mathrm{HCl}$ to a $\mathrm{pH}$ of 4-5, as determined using universal indicator strips, and then extracted with EtOAc $(3 \times 150 \mathrm{~mL})$. The combined extracts were washed with water $(2 \times 50 \mathrm{~mL})$ then dried $\left(\mathrm{MgSO}_{4}\right)$, and concentrated under reduced pressure to afford compound 9 (350 $\mathrm{mg}$, $1.23 \mathrm{mmol}, 74 \%$ ). This crude sample was identical, as judged by ${ }^{1} \mathrm{H}-\mathrm{NMR}$ and low resolution mass spectrometric analysis with previously reported authentic material. Due to the previously reported limited stability of this compound it was immediately subjected to the following hydrogenation step. ${ }^{6}$

(R)-2-Aminomethyl-3-phenylpropionic acid (10). The crude sample of compound 9 (350 mg, $1.23 \mathrm{mmol}$ ) from the previous step, was dissolved in $\mathrm{MeOH}, \mathrm{NH}_{3}$ and $\mathrm{H}_{2} \mathrm{O}(3: 1: 1,10 \mathrm{~mL})$ and $10 \% \mathrm{Pd} / \mathrm{C}(100 \mathrm{mg})$ was added to the solution. The mixture was then stirred at RT under an atmosphere of hydrogen for $72 \mathrm{~h}$ (at which point TLC analysis indicated complete consumption of starting material), before being filtered through a plug of Celite, and concentrated under reduced pressure, to provide compound 10. This crude sample was identical, as judged by ${ }^{1} \mathrm{H}-$ NMR with previously reported authentic material. ${ }^{8,9}$

(R)-2-[(9H-Fluoren-9-ylmethoxycarbonylamino)methyl]-3-phenylpropionic acid (11). The crude sample of compound $\mathbf{1 0}(350 \mathrm{mg})$ produced in the previous step was dissolved in THF and $\mathrm{H}_{2} \mathrm{O}\left(10 \mathrm{~mL}\right.$ of a 1:1 mixture) and cooled to $0{ }^{\circ} \mathrm{C}$, whilst being stirred under nitrogen. The solution was treated with Fmoc-OSu $(0.420 \mathrm{~g}, 1.25 \mathrm{mmol})$ and $\mathrm{NaHCO}_{3}(1.0 \mathrm{~g}, 11.88 \mathrm{mmol})$ and allowed to warm to RT over the course of $2 \mathrm{~h}$. THF was removed under reduced pressure and the aqueous residue was washed with diethyl ether $(2 \times 10 \mathrm{~mL})$. The organic layers were discarded and the aqueous layer was acidified with sat. aq. $\mathrm{KHSO}_{4}$ and extracted with DCM (3 x $20 \mathrm{~mL})$. The combined organic extracts were dried $\left(\mathrm{MgSO}_{4}\right)$, filtered, and concentrated under reduced pressure. The residue was purified via flash chromatography. Concentration of the appropriate fractions $\left(R_{\mathrm{f}}=0.3,50: 1 \mathrm{DCM} / \mathrm{MeOH}\right)$ afforded compound 11 (122 $\mathrm{mg}, 0.36 \mathrm{mmol}$, $\sim 30 \%$ over two steps, $>95 \%$ e.e. as determined by chiral HPLC). This sample of compound 11 was identical, as judged by ${ }^{1} \mathrm{H}-\mathrm{NMR}$, low resolution mass spectrometric analysis and chromatographic retention characteristics with previously reported authentic material. ${ }^{8,9}$ Anal. Calcd for $\mathrm{C}_{25} \mathrm{H}_{23} \mathrm{NO}_{4}$ : C, 74.80; H, 5.77; N, 3.49\% Found: C, 74.63; H, 5.77; N, 3.45\% 


\section{References}

1. Hein, J. E.; Hultin, P. G. Synlett 2003, 5, 635.

2. Hein, J. E.; Geary, L. M.; Jaworski, A. A.; Hultin, P. G. J. Org. Chem. 2005, 70, 9940.

3. Hein, J. E.; Zimmermann, J.; Sibi, M. P.; Hultin, P. G. Org. Lett. 2005, 7, 2755.

4. Brenner, M.; La Vecchia, L.; Leutert, T.; Seebach, D. Org. Synth. 2003, 80, 57.

5. Beddow, J. E.; Davies, S. G.; Ling, K. B.; Roberts, P. M.; Russell, A. J.; Smith, A. D.; Thomson J. E. Org. Biomol. Chem. 2007, 5, 2812.

6. Slade, J.; Parker, D.; Girgis, M.; Mueller, M.; Vivelo, J.; Liu, H.; Bajwa, J.; Chen, G.-P.; Carosi, J.; Lee, P.; Chaudhary, A.; Wambser, D.; Prasad, K.; Bracken, K.; Dean, K.; Boehnke, H.; Repic, O.; Blacklock, T. J. Org. Process Res. Dev. 2006, $10,78$.

7. Pratt, L. M.; Beckett, R. P.; Davies, S. J.; Launchbury, S. B.; Miller, A.; Spavold, Z. M.; Todd, R. S.; Whittaker, M. Bioorg. Med. Chem. Lett. 2001, 11, 2585.

8. Guichard, G.; Abele, S.;Seebach, D. Helv. Chim. Acta 1998, 81, 187.

9. Lee, H.-S.; Park, J.S.; Kim, B. M.; Gellman, S. H. J. Org. Chem. 2003, 68, 1575.

10. Nicolaou, K. C.; Webber, S. E. Synthesis 1986, 453.

11. Bayardon, J.; Cavazzini, M.; Maillard, D.; Pozzi, G.; Quici, S.; Sinou, D. Tetrahedron: Asymmetry 2003, 14, 2215.

12. Alvey, L. J.; Rutherford, D.; Juliette, J. J. J.; Gladysz, J. A. J. Org. Chem. 1998, 63, 6302.

13. Teeter, H. M.; Bell, E. W. Org. Synth. 1963, 4, 125.

14. Zhang, W.; Curran, D. P. Tetrahedron 2006, 62, 11837.

15. Zhang, W. F. QSAR Comb. Sci. 2006, 25, 679. 